\title{
TÉCNICAS Y SISTEMAS ACTUALES DE OBTURACIÓN EN ENDODONCIA. REVISIÓN CRÍTICA DE LA LITERATURA
}

\author{
CURRENT TECHNIQUES AND SYSTEMS OF OBTURATION IN \\ ENDODONTICS. CRITICAL REVIEW OF LITERATURE
}

\author{
Andrea Gisella Flores - Flores ${ }^{1 \mathrm{a}, 2 \mathrm{~b}}$, Aissa Pastenes - Orellana ${ }^{3 a, 4 b}$
}

\section{RESUMEN}

Debido a la compleja anatomía radicular, existe una búsqueda constante de un sellado tridimensional lo más herméticamente posible en la obturación posibilitando la reparación de los tejidos. Desde su descubrimiento, la gutapercha sigue siendo el material ideal para este propósito, debido a que posee la particularidad de adecuarse a los diferentes cambios térmicos y sus desventajas no hacen menguar sus cualidades.

Esta revisión crítica de la literatura tiene como objetivo enfatizar conceptos y destacar aspectos positivos y vulnerables de las diferentes técnicas y sistemas de obturación con la finalidad de potenciar al máximo un correcto sellado homogéneo, cumpliendo con nuestros objetivos técnicos y biológicos.

Se empleó el método cualitativo, análisis de contenido de tipo directo, realizándose una búsqueda exhaustiva de la información, concluyéndose que la técnica o sistema que se emplee dependerá de los conocimientos, destrezas y recursos disponibles teniendo en cuenta las características anatómicas a las que el clínico se enfrente. La mayoría de los sistemas que utilizan gutapercha termoplastificada son de costo elevado dificultando su utilización en la práctica diaria endodóncica. La hibridación de las diferentes técnicas y sistemas permite obtener un sellado lo más homogéneo posible.

Palabras Clave: Endodoncia; Obturación del conducto radicular; Gutapercha; Materiales de obturación del conducto radicular. (Fuente: DeCS BIREME)

\section{ABSTRACT}

Due to the complex radicular anatomy, there is a constant search for a three - dimensional seal - as tight as possible in the obturation - making possible the repair of the tissues. Since its discovery, gutta-percha remains as the ideal material for this purpose; because it has the particularity of adapting to the different thermal changes and its disadvantages do not diminish its qualities. This critical review of the literature aims to emphasize concepts and highlight positive and vulnerable aspects of the different techniques and sealing systems, with the aim of maximizing a correct homogenous sealing, fulfilling our technical and biological objectives.

The qualitative analysis method of direct type content was used; an exhaustive search of the information is carried out, concluding that the technique or system used will depend on the knowledge, skill and available resources, taking into account the anatomical characteristics to which the clinician is confronted. Most systems that use thermoplasticized gutta- percha are expensive and difficult to use in endodontic daily practice. The hybridization of the different techniques and systems allows a seal to be obtained as homogeneous as possible.

Keywords: Endodontic; Root canal obturation; Gutta-percha; Root canal filling materials. (Source: MeSH NLM)

Recibido: 02 de mayo 2018

Aprobado: 25 de junio 2018

Publicado: 30 de junio 2018

${ }_{1}^{1}$ Universidad de San Martin de Porres. Lima, Perú

2 Universidad Inca Garcilaso de la Vega. Lima, Perú.

${ }^{3}$ Universidad de Chile. Chile.

${ }^{4}$ Universidad Peruana Cayetano Heredia. Lima, Perú.

${ }^{a}$ Cirujano dentista, ${ }^{b}$ Especialista en Endodoncia

Correspondencia:

Andrea Flores

Correo electrónico: andreaflores30@hotmail.com

Citar como: Flores - Flores A., Pastenes - Orellana A. Técnicas y sistemas actuales de obturación en endodoncia. Revisión crítica de la literatura. KIRU. 2018 abril-jun; 15(2): 85-93. doi.org/10.24265/kiru.2018.v15n2.05
Este es un artículo Open Access distribuido bajo la licencia Creative Commons Atribución-NoComercial- Compartir Igual 4.0

\section{(C) $(9)$}




\section{INTRODUCCIÓN}

La obturación endodóncica es la última fase del tratamiento de conductos radiculares y no por ello la menos importante. Está demostrado que la mayoría de fracasos endodóncicos se relacionan con obturaciones deficientes, es decir, aquellas que no cumplen los principios básicos de una óptima obturación ${ }^{(1,2)}$.

Una vez culminada la preparación químico-quirúrgica se debe proceder a obturar el sistema de conductos radiculares con materiales biocompatibles, inertes $\mathrm{y} / \mathrm{o}$ antisépticos. El objetivo primordial es alcanzar una obturación lo más herméticamente posible, con un sellado tridimensional que impida el estancamiento de fluidos y la supervivencia de microorganismos para la posterior reparación de los tejidos, evitando así una reinfección ${ }^{(3,4)}$.

Las consideraciones al momento de obturar con respecto al límite apical son las mismas que se aplicaron en la preparación de los conductos radiculares y que fueron establecidas por la conductometría. Recordemos que debemos situarnos lo más cercanos al límite del conducto radicular y cementario, llamado límite CDC o campo de acción del endodoncista ${ }^{(5,6,7)}$.

La evolución de técnicas y sistemas de obturación en estas dos últimas décadas es considerada un gran avance en cuanto a simplificación, perfeccionamiento y sellado homogéneo del sistema de conductos. La técnica o sistema que empleemos dependerá de nuestros conocimientos, destreza, recursos y materiales, así como también de la intrincada y variada anatomía de conductos radiculares en la que estemos trabajando ${ }^{(8.9,10)}$.

Esta recopilación crítica de información actualizada tiene como objetivo enfatizar conceptos y destacar aspectos positivos y vulnerables de las diferentes técnicas y sistemas de obturación. Se empleó el método cualitativo, análisis de contenido de tipo directo. La revisión de información se basó en la búsqueda de información en revistas indexadas en Medline, Scielo, Pubmed, Embase, Lilacs; con la utilización de descriptores como "endodoncia", "obturación del conducto radicular", "gutapercha", "materiales de obturación del conducto radicular". Los criterios de inclusión correspondieron a los idiomas español, inglés y portugués. Se consideraron artículos de cualquier área geográfica o país preferentemente publicado en los últimos cinco años y se consideró también la búsqueda manual de libros y revistas. Los criterios de exclusión fueron: casos clínicos einformes.
La búsqueda tuvo la finalidad de permitir al clínico la mejor elección en cuanto a técnicas y sistemas de obturación para lograr un sellado lo más homogéneo posible, basándose en la evidencia científica.

\section{Objetivos de la obturación:}

Objetivo técnico: Conseguir un relleno lo más hermético posible de la totalidad del sistema de conductos radiculares, sin sobrepasar los límites preestablecidos, no alcanzando al periodonto $(2,7)$.

Objetivo biológico: Es la reparación de los tejidos. Al no llegar productos tóxicos al periápice, los propios medios de defensa del organismo podrán, por lo general, eliminar las bacterias, componentes antigénicos y restos hísticos necróticos que hayan quedado junto al ápice ${ }^{(2)}$, como también generar aposición de cemento en las zonas reabsorbidas ${ }^{(7,11)}$.

Los principios básicos antes de proceder a obturar son la irrigación y aspiración al término de la instrumentación. Consisten en irrigar con hipoclorito de sodio al $1 \%$, luego con EDTA $17 \%$, seguido de hipoclorito de sodio $1 \%$ (cerca de $5 \mathrm{ml}$ de cada solución por conducto). Este protocolo promueve una mejor limpieza que el solo uso de hipoclorito de sodio.

La activación ultrasónica es altamente recomendable (2,5 $\mathrm{ml}$ de EDTA 17\% + activación ultrasónica por 30 segundos $+2,5 \mathrm{ml}$ de hipoclorito de sodio al $1 \%$ ), ya que proporciona una limpieza considerable de las paredes dentinarias ${ }^{(12)}$.

\section{Propiedades ideales de un material obturador:}

Debe ser radiopaco, antimicrobiano, insoluble a líquidos tisulares, biocompatible; que no irrite los tejidos periapicales promoviendo su cicatrización y reparación.

Debe tener fluidez y capacidad de adaptación a las paredes de los conductos radiculares; gran estabilidad dimensional, así como facilidad para ser manipulado y retirado en casos de retratamientos o preparaciones para la colocación de retenedores intrarradiculares. No debe ser corrosivo ni oxidante, no debe teñir la dentina $\mathrm{y}$, por último, debe ser un material de bajo costo ${ }^{(5,7,13)}$.

En la actualidad existen materiales de obturación que no satisfacen todos estos criterios, sin embargo, es la gutapercha el material de elección. Se han propuesto diversas alternativas para reemplazarla, pero ningún otro material ha mostrado estar en condiciones de sustituirla. La gutapercha permanece como el "Gold Standard" para la obturación endodóncica $(5,7,13)$. 


\section{GUTAPERCHA:}

En el año 1867, se introduce la gutapercha en endodoncia como material obturador de conductos radiculares. Es una sustancia vegetal extraída en forma de látex de los árboles de la familia de las sapotáceas (Mimusops balata y Mimusops luben). Esta sustancia existente en Sumatra, Filipinas, Archipiélago Malayo y Brasil se combina con otros materiales para formar una mezcla que puede utilizarse con efectividad dentro del conducto $(12,14,15,16)$.

\section{Propiedades principales:}

- Termoplasticidad: permite trabajar con los conos de gutapercha en estado caliente.

- Viscoelasticidad: es la propiedad principal de la gutapercha; esta sufre una deformación plástica cuando es sometida a una fuerza de condensación por un breve lapso; de la misma forma admite trabajar con conos de gutapercha en estado frío ${ }^{(17,}$ 13,12).

\section{Ventajas de la gutapercha:}

Es un material radiopaco, biocompatible, con buena tolerancia tisular e insoluble a líquidos orgánicos. Posee estabilidad dimensional y es un material de fácil desobturación ${ }^{(12,18)}$. Tiene una aceptable adaptación a las paredes del conducto radicular, capacidad de ablandamiento y plastificación por medio del calor y disolventes orgánicos. Posee, además, estabilidad física y química ${ }^{(6,17)}$, y es el material disponible menos alergénico ${ }^{(13,17,19)}$.

\section{Desventajas de la gutapercha:}

Escasa rigidez, dificultando su uso en conductos curvos. Podría ser movida por presión, causando sobre obturación durante los procesos de condensación ${ }^{(7,19)}$. Existen una diversidad de técnicas para plastificar la gutapercha, no obstante, todas ellas precisan el recurso de un cemento para un mejor sellado $(2,7,12)$.

\section{Fases de la gutapercha:}

Comprende dos formas esteáricas cristalinas que son la fase alfa ( $\alpha$ ) y la fase beta $(\beta)$. Existe también una forma amorfa o fundida. Las tres forman parte de la obturación de conductos radiculares ${ }^{(6,12)}$.

Las puntas o conos convencionales de gutapercha están fabricadas en fase beta $(\beta)$, que se transforma en fase alfa $(\alpha)$ cuando se calienta a 42 y $49^{\circ} \mathrm{C}$, volviéndose más estable, más fluida y moldeable. Si se calienta de 53 a $59^{\circ} \mathrm{C}$ se pierde el estado semicristalino alfa (a) para proporcionar una mezcla amorfa a $59^{\circ} \mathrm{C}$ $(5,12,16)$.

\section{Composición y presentación de los conos de gutapercha:}

- Componentes orgánicos:

Gutapercha $19-21 \%$, ceras $1,4 \%$, resinas $1,4 \%$, colorantes $1,4 \%$.

- Componentes inorgánicos:

Óxido de Zinc, 59-75\%; sales de bismuto, 1,17\%; sulfato de estroncio, $1,17 \%$. Algunos contienen sulfato de cadmio, $1,17 \%^{(7,12,19)}$.

Se presentan conos estandarizados y no estandarizados. Estos últimos son de diferentes conicidades y corresponden a los instrumentos de los sistemas mecanizados. Tienen, además, la ventaja de facilitar la obturación al requerir una menor cantidad de conos accesorios, haciendo posible incluso la obturación con un solo cono principal bien adaptado. Actualmente algunas marcas comerciales presentan una mayor cantidad de óxido de zinc en su composición haciendo que los conos principales no estandarizados sean más rígidos ${ }^{(7)}$.

\section{TÉCNICAS DE OBTURACIÓN:}

\section{- Técnica de condensación lateral en frío:}

Inicialmente propuesta en 1914, su eficacia comprobada, relativa sencillez, facilidad en el control del límite apical y el empleo de un instrumental simple hacen que sea la técnica más utilizada. Es considerada una técnica patrón cuya eficacia se compara con otras técnicas más novedosas ${ }^{(7,14,18)}$.

Con la intención de mejorar ampliamente nuestro objetivo de sellado tridimensional y respetando todos los principios de una óptima obturación y consecuente reparación de los tejidos, es que se proponen nuevas técnicas alternativas como las ejecutadas a base de gutapercha caliente. El reblandecimiento mediante calor tiene como finalidad optimizar su adaptación a las irregularidades de la anatomía interna de los conductos radiculares $(7,13,18)$.

\section{- Técnica de condensación vertical de gutapercha caliente:}

Diseñada por Shilder, esta técnica cambió radicalmente los conceptos de obturación estableciendo un gran debate y discusión en los años setenta, puesto que para ese entonces la condensación lateral de gutapercha en 
frío acompañada por solventes era la más popular ${ }^{(12)}$. Consiste en la adaptación de un cono no estandarizado como Medium-Fine o Fine-Medium o actualmente puntas de gutapercha con conicidades continuas como $4 \%$ ó $6 \%$. Los condensadores seleccionados pueden ser los creados por Shilder, más actuales y simplificados de níquel-titanio como los de Buchanan, o los de Thompson (12).

La primera fase es denominada Downpack; corresponde a la obturación del tercio apical del conducto radicular en sentido corono-apical ${ }^{(12)}$.

Se introduce el cono principal de gutapercha con cemento en el interior del conducto, seccionándolo con calor y empacándolo verticalmente con el condensador de mayor grosor, obteniendo una distribución de la gutapercha y del cemento en tres dimensiones a lo largo del conducto en un segmento de 4-5mm, también en los conductos laterales $(7,16)$.

Durante el último proceso de calentamiento, el espaciador térmico alcanza la zona apical y el condensador más delgado se introduce hasta máximo

$5 \mathrm{~mm}$ de la constricción apical. En esta fase, la condensación obtura pequeñas ramificaciones y posibles deltas apicales. La introducción se realiza con una fuerte presión dirigida hacia el tercio apical hasta que se enfríe la gutapercha para evitar una contracción durante la fase de enfriamiento ${ }^{(20)}$. Es importante obtener una radiografía para asegurar una obturación de calidad apical ${ }^{(12,21)}$.

Se recomienda calentar la gutapercha con aparatos especiales, como por ejemplo Touch $\mathrm{N}$ Heat (Sybron Endo), System B (Sybron Endo) Down Pak (Hu-Friedy), entre otros, permitiendo plastificarla por segmentos ${ }^{(12)}$.

En esta segunda etapa, se adapta un retenedor intrarradicular o se procede a completar la obturación del espacio que resta en sentido ápico coronal (Backfill) $(12,16)$, correspondientes a los tercios medio y cervical. Para ello, se emplea una pistola de gutapercha, como por ejemplo el Sistema Obtura II (SybronEndo, Orange, CA, EUA), o el Sistema Obtura III - Calamus Dual (Dentsply Tulsa Dental). Ambos utilizan una cánula de inyección que calienta la gutapercha condensada apicalmente permitiendo además una buena adherencia de la gutapercha condensada $(12,20)$.

\section{- Técnica de condensación vertical de onda continua:}

Desarrollada por Buchanan, es una evolución de la técnica de condensación vertical de gutapercha caliente. Se basa en la utilización de un transportador de calor eléctrico System $B \Theta$ (SybronEndo, Orange,
CA. EUA) con diversas puntas flexibles en diferentes conicidades, de $4 \%, 6 \%, 8 \%, 10 \%$ y $12 \%$. En el monitor del equipo se muestra la temperatura en la cual se calentará la gutapercha $\left(200^{\circ} \mathrm{C}+,-10^{\circ} \mathrm{C}\right){ }^{(12)}$ y con la ayuda de una pieza de mano con una punta transportadora de calor se calienta la gutapercha y luego se condensa. Este sistema utiliza conos de gutapercha no estandarizados en fase alfa $(\alpha)^{(7,12,13)}$.

La técnica se realiza aplicando previamente cemento sellador en las paredes del conducto radicular y luego se lleva el cono maestro a la longitud de trabajo. Con la pieza de mano caliente se realiza una leve presión de condensación vertical hasta llegar a 3-4 mm de la longitud de trabajo. Luego se desactiva el calor de la punta del condensador para continuar la condensación vertical con la punta fría. Seguidamente, se eleva la temperatura del monitor a $300^{\circ} \mathrm{C}$ y se activa la punta por un segundo para poder sacar la punta del condensador del conducto sin extraer la gutapercha que se había compactado ${ }^{(7,12)}$.

La fase de remoción de la gutapercha en los segmentos medio y cervical es denominada downpack y puede ser realizada también con otro aparato, el Touch'N Heat (SybronEndo, Orange, CA, EUA).

Una vez obturado el tercio apical, se procede a terminar la obturación con más aplicaciones de gutapercha en los tercios medio y cervical (llamada fase backfill), con sistemas de inyección, como por ejemplo el Sistema Obtura II, o Sistema Obtura III - Calamus Dual (Dentsply Tulsa Dental) ${ }^{(13,21)}$.

Gupta et al. compararon la técnica de condensación lateral versus los sistemas Thermafil \& (Dentsply Maillefer) y Calamus Dual (Dentsply Tulsa Dental). Utilizaron tomografías de haz cónico para medir las áreas de gutapercha y los espacios vacíos en los tercios coronal, medio y apical. Observaron mayor cantidad de material obturador en el grupo del sistema Calamus Dual (Dentsply Tulsa Dental) seguido del sistema Thermafil @ (Dentsply Maillefer) ${ }^{(22)}$.

\section{Consideraciones importantes:}

En conductos curvos, el ingreso de los instrumentos hasta la profundidad deseada es más difícil, siendo necesaria una mayor preparación de los segmentos cervical y medio pudiendo causar un debilitamiento de las paredes radiculares ${ }^{(22)}$.

Existe una mayor dificultad en el tratamiento de piezas dentarias de gran longitud por una difícil adaptación en niveles deseados, siendo inviable la utilización de esta 
técnica ${ }^{(24,20)}$. La gutapercha reblandecida por el calor y condensada al mismo tiempo logra penetrar espacios muy pequeños como istmos, conductos laterales, deltas apicales, etc ${ }^{(12)}$.

Otros sistemas que realizan la misma función: Beefill 2 in 1 (VDW), Obtura III - Calamus Dual, Downpack e Backfill (Dentsply Tulsa Dental), Sistema EQ Plus (Driller, São Paulo, Brasil) presentan una interesante combinación de funciones: el principio de calor por onda continua con la asociación de una inyección de gutapercha termoplastificada ${ }^{(13)}$.

\section{- Técnicas de inyección de gutapercha termoplastificada:}

Es una variación de las técnicas termoplastificadas. Se emplea un cemento sellador de conductos colocándolo previamente en las paredes del conducto radicular con una lima o puntas de papel (12).

Los sistemas Obtura II (Obtura Corporation), Obtura III - Calamus Dual (Dentsply Tulsa Dental), Elements Obturation (SybronEndo), HotShot (SybronEndo), Sistema Ultrafilß 3D (Coltene Whaledent, Cuyahoga Falls, EUA), System B Cordless (SybronEndo), Beefell 2 in 1 (VDW), Sistema EQ Plus (Driller, São Paulo, Brasil), actúan bajo el mismo principio de inyección de gutapercha termoplastificada $^{(7,12)}$.

\section{- $\quad$ Sistema Obtura II (Obtura Corporation):}

Utiliza un horno para calentar la gutapercha fase beta $(\beta)$ contenida en una jeringa tipo pistola, hasta alcanzar temperaturas que oscilan entre los $160^{\circ} \mathrm{C}$ y los $200^{\circ} \mathrm{C}$. La gutapercha caliente es expulsada por presión desde la jeringa hasta el conducto a través de agujas calibre 20 ó 23. Esta gutapercha es liberada de la cánula a una temperatura entre $47^{\circ}$ y $81^{\circ} \mathrm{C}$, como máximo, sin producir lesiones en el ligamento periodontal marginal adyacente. Este sistema ha sido criticado por las altas temperaturas a la que es sometida la gutapercha cuando entra en contacto con el conducto radicular; sin embargo, no se han demostrado daños permanentes en los tejidos perirradiculares. Es un sistema de muy alto costo. Actualmente contamos también con el Sistema Obtura III - Calamus Dual (Dentsply Tulsa Dental) $(7,12)$.

\section{Aplicación de la gutapercha sin condensación vertical:}

Medir la aguja, colocarla a $3 \mathrm{~mm}$ de la longitud de trabajo, aplicar el cemento sellador aproximadamente a la misma longitud a la que debe entrar la aguja de inyección. Si se lleva a la longitud total de trabajo, se podría sobrepasar al periápice en el momento de la inyección; luego colocar la temperatura en el equipo entre $185^{\circ}$ y $200^{\circ} \mathrm{C}$ posicionando la aguja dentro del conducto radicular e inyectando lentamente la gutapercha. En este proceso se debe evitar la presión apical con la aguja. Durante su aplicación, la masa inyectada empujará suavemente la aguja hacia la porción coronal del conducto y se seguirá inyectando gutapercha incrementalmente hasta obturar por completo el conducto radicular. Es importante recordar que la preparación apical debe ser lo más estrecha posible para evitar sobreobturaciones $(12,13)$.

\section{Variación de la técnica:}

Se puede realizar una condensación lateral del tercio apical, recorte de la gutapercha a este nivel y luego inyectar gutapercha termoplastificada con el sistema obtura ${ }^{(13)}$.

\section{- $\quad$ Sistema Ultrafilß 3D (Coltene Whaledent, Cuyahoga Falls, EUA):}

Es un sistema de inyección de gutapercha termoplastificada de baja temperatura que se compone de una jeringa de inyección. Esta última es cargada con cánulas de gutapercha que son previamente entibiadas en un horno especial. Para este sistema, es posible elegir entre tres tipos de gutapercha que son: la serie sólida (cánula azul) que endurece de forma más rápida la gutapercha; la serie regular (cánula blanca) que presenta una menor contracción al enfriarse; y la serie Endoset $\AA$ con un perfil de solidificación mixto. La salida de la aguja tiene un diámetro equivalente a una lima número $70^{(7,12,13)}$.

\section{- Técnicas de gutapercha termoplastificada con núcleo sólido:}

\section{- $\quad$ Sistema Thermafilß (Dentsply Maillefer):}

Son vástagos plásticos estandarizados recubiertos de gutapercha en fase alfa ( $\alpha$ ). Utiliza un horno especial que al calentar los vástagos reblandecen la gutapercha permitiendo obturar el conducto radicular en un solo paso.

Los conductos unirradiculares y de gran calibre deben ser instrumentados aproximadamente hasta una lima número 60 ó 70 para garantizar un buen sellado y manejo del sistema de obturación.

Se debe aplicar cemento sellador al momento de introducir este sistema luego se esperan 3 minutos y con una fresa de carburo larga se secciona la punta del Thermafil en la entrada del conducto $(12,25,26,27)$. 
Samadi $\mathrm{F}$ et al. realizaron un estudio in vitro sobre la eficacia de tres técnicas de obturación. Evaluaron el porcentaje del área de obturación con gutapercha: grupo I, sistema Thermafilß (Dentsply Maillefer); grupo II, condensación vertical y grupo III, condensación lateral. Bajo la fotografía digital y la estereomicroscopía procedieron a analizar las secciones transversales del tercio apical de cada grupo. Se encontraron diferencias estadísticamente significativas: una mejor área de obturación con gutapercha en el grupo I, seguida por el grupo II y finalmente el grupo III (28).

\section{- Otros sistemas:}

Quik-fill (JS Dental), compuesto por núcleos de titanio envueltos de gutapercha en fase alfa $(\alpha)^{(6)}$. MicroMega® (HEROfill $\left.{ }^{\circledR}\right)$ con núcleos de plástico todos recubiertos con una capa de gutapercha en fase alfa (a). GT Obturators (Dentsply/Maillefer), similar al sistema Thermafil, presentando núcleos de plástico ${ }^{(6)}$. Soft-Core $^{\mathrm{TM}}$ (SybronEndo), gran flexibilidad para desplazarse en conductos curvos. Sus núcleos son más delgados que los de Thermafil, lo cual permite una mayor flexibilidad utilizando; además, un solo tamaño de conicidad universal ${ }^{(29)}$.

Una de las desventajas principales es la presencia de núcleos sólidos compuestos de plástico o metal que impiden desobturar la gutapercha en casos de retratamiento o en la colocación de un retenedor intrarradicular. Por esta limitación se introdujo un nuevo material que reemplaza el núcleo de plástico por un vástago de gutapercha entrelazada de gran resistencia, llamado GuttaCore (Dentsply Tulsa Dental Specialities) o GuttaFusion (VDW). Ambos desarrollaron el mismo principio, brindando una obturación de gutapercha caliente hasta el ápice, sin burbujas y con un ajuste fiable y seguro $(20,30,31)$.

También es posible encontrar otros sistemas como: McSpadden II ${ }^{\mathrm{TM}}$, Alphaseal ${ }^{\mathrm{TM}}$ (NiTi Co., Chattanooga, EUA) y Successfill ${ }^{\mathrm{TM}}$ (Coltene Whaledent). Los tres poseen características muy semejantes. La gutapercha se prepara en una jeringa y es calentada en un horno. Posteriormente, es introducida en el conducto a través de limas o de termocondensadores. Difieren entre sí por la composición de la gutapercha, siendo el Successfill $^{\mathrm{TM}}$ y el Alphaseal ${ }^{\mathrm{TM}}$ muy parecidos a la gutapercha en la fase alfa $(\alpha)$.

Por otra parte, McSpadden //TM utiliza dos tipos de gutapercha, en la que se introduce el compactador de la gutapercha fase beta (b) para dar cuerpo a la obturación y posteriormente en la fase alfa ( $\alpha$ ), para permitir un mejor escurrimiento y adhesividad ${ }^{(12)}$.
- Técnica de termocompactación de la gutapercha:

Introducida en 1980 por John McSpadden, por medio de Ransom \& Randolph, esta técnica consiste en compactar y plastificar la gutapercha dentro del conducto radicular mediante la acción mecánica de instrumentos apropiados -similares a una lima Hedström invertida- estandarizados y adaptados a un contrángulo de baja velocidad girando en sentido horario ${ }^{(12)}$.

Sin embargo, la adaptación del cono de gutapercha principal, seguido de la acción del termocompactador promovía una frecuente extravasación perirradicular haciendo inviable su aplicación clínica, (13) como también el calentamiento desigual de la gutapercha y la fractura de los instrumentos ${ }^{(11)}$. Estos problemas fueron eludidos posteriormente por Tagger et al. estudiaron in vitro el sellado apical producido por la asociación de la técnica de condensación lateral en combinación con el compactador termomecánico, dando origen a las siguientes técnicas ${ }^{(7)}$ :

- Técnica híbrida de Tagger:

Se basa en condensar lateralmente a nivel apical, con un espaciador digital fino, el cono principal de gutapercha. Se encaja en el espacio abierto por él un cono auxiliar y entre los conos de gutapercha se introduce el compactador termomecánico Engine Plugger (el instrumento se asemeja a una lima tipo $\mathrm{k}$ invertida). Este último compactador se utiliza en un contrángulo de baja rotación combinando la condensación lateral activa en frío a nivel apical, seguida de la termocompactación en los tercios medio y cervical ${ }^{(7)}$.

\section{- Técnica híbrida modificada:}

Posteriormente Tagger et al. propusieron una modificación en la técnica original, la cual consiste en realizar inmediatamente después de la utilización del termocompactador un nuevo espaciamiento lateral seguido de la inserción de uno o dos conos de gutapercha, reutilizándose entonces el termocompactador con el objetivo de mejorar la densidad de la obturación. Esta maniobra también puede ser utilizada en la corrección de otras técnicas de obturación, especialmente en la eliminación de porosidades y en el relleno denso y homogéneo de canales con curvaturas acentuadas y ramificaciones en la región apical.

Además, esta técnica reduce significativamente el tiempo operatorio en un $40 \%$, en comparación con la 
técnica de compactación lateral ${ }^{(13,32)}$.

Se utilizan compactadores semejantes a una lima Hedström invertida Gutta Condensor ${ }^{\circledR}$ (Maillefer), semejantes a los de Ransom \& Randolph (7). Actualmente se fabrican también instrumentos con aleación de níquel-titanio basados en el mismo principio como el Gutta Condensor $^{\circledR}$ (Maillefer) $^{(12)}$.

\section{- Sistema de obturación Microseal:}

Analytic Endodontics (Glendora, EUA) lanzó el Sistema Microseal de obturación, compuesto por un horno con jeringa que permite calentar cartuchos con gutapercha, un contrángulo, conos de gutapercha de baja fusión (conos principales) y conos de ultra baja fusión (colocados en cartuchos y jeringa). Estos últimos conos son utilizados con la técnica de termoplastificación mediante la utilización de espaciadores y compactadores fabricados de aleación de níquel-titanio ${ }^{(7,13)}$.

Se empieza adaptando el cono principal de baja fusión envuelto en cemento y se inserta un espaciador de níquel-titanio para realizar una condensación lateral. Este procedimiento permite dejar un espacio que será ocupado por gutapercha de ultra baja fusión, la cual fue calentada previamente en un cartucho dentro de un calefactor adaptado en una jeringa especial y retirada mediante un compactador de níquel-titanio. El tiempo de trabajo promedio es de 30 segundos. Sin aplicar presión apical alguna, pero resistiendo al reflujo del material obturador, se debe comenzar la rotación del compactador a una velocidad de 5000 a 7000 rpm. Después, por no más de 2 segundos, empezar lentamente a retirar el compactador, aplicando una suave presión en uno de los lados del conducto radicular (no parar la rotación hasta que el compactador esté completamente fuera del conducto y no superar los 6 segundos de tiempo en la compactación). Este sistema posee la ventaja de corregir la obturación cuantas veces sea necesaria, evitando la remoción de todo el material obturador. Además, es una alternativa de bajo costo en relación a otros sistemas ${ }^{(7)}$.

\section{- Técnica de obturación con ultrasonido}

Permite efectuar una obturación termoplastificada controlando la plastificación de la gutapercha y, consecuentemente, obteniendo una obturación más hermética y uniforme en toda la extensión del conducto radicular.

La punta de ultrasonido indicada tiene un soporte para colocar el espaciador y condensador y para el corte de los excesos de gutapercha se emplea otra punta curva en forma de hoz ${ }^{(33)}$.

Después de la colocación del cono principal, se realiza una secuencia de la técnica de condensación lateral activa, empleando el espaciador ultrasónico.

Se introduce el espaciador ultrasónico lateralmente al cono de gutapercha principal con presión apical -sin accionar el aparato de ultrasonido- hasta que el instrumento alcance una resistencia a la penetración cercana al tope apical. Seguidamente se acciona el ultrasonido durante 10 segundos con movimientos laterales para ampliar progresivamente el espacio, se desactiva el ultrasonido y se remueve el espaciador lentamente sin alterar la posición del cono principal y de los auxiliares; luego llevar al espacio obtenido un cono auxiliar de gran tamaño y conicidad alrededor del cemento obturador buscando alcanzar la misma profundidad de introducción del instrumento ${ }^{(7)}$.

Siu et al. realizaron un estudio en el que compararon in vitro la densidad en la obturación de la gutapercha de tres diferentes técnicas: condensación lateral, obturación con ultrasonido y compactación vertical con gutapercha caliente. No emplearon cemento obturador, se tomaron tomografías computarizadas a los treinta y tres primeros molares inferiores antes y después de la obturación. Concluyeron que la compactación vertical con gutapercha caliente y la obturación con ultrasonido presentaron una mayor densidad comparada con la condensación lateral ${ }^{(34)}$.

\section{CONCLUSIONES}

- La técnica o sistema de obturación que se emplee dependerá de los conocimientos, destreza y recursos disponibles, teniendo en cuenta las características anatómicas a la que se enfrente con el objetivo de conseguir una obturación lo más tridimensionalmente posible. En situaciones adversas como reabsorciones radiculares internas, conductos muy amplios o conductos en "C", se recomienda la utilización de gutapercha termoplastificada o termocompactada ya que presenta un sellado más homogéneo obteniendo mejores resultados en estos casos.

- El costo elevado de la mayoría de los sistemas que utilizan gutapercha termoplastificada dificulta su utilización en la práctica diaria endodóncica.

- La hibridación de las diferentes técnicas y sistemas es una alternativa que permite obtener un sellado lo más homogéneo posible de acuerdo a las condiciones anatómicas. 


\section{REFERENCIAS BIBLIOGRÁFICAS}

1. Hargreaves K, Bernan L, Cohen S. Vías de la pulpa. 11를 ed. Barcelona: Elsevier; 2016.

2. Canalda S, Brau A. Endodoncia: Técnicas Clínicas y Bases científicas. $3^{\underline{a}}$ ed. Madrid: Editorial Elsevier Masson; 2014.

3. Vieira $R$, Ferreira $F$, Campolina $M$, Húngaro $M$, Cavalini B, Gomes I et.al. Filling Effectiveness and Dentinal Penetration of Endodontic Sealers: A Stereo and Confocal Laser Scanning Microscopy Study. Brazilian Dental Journal. 2015 Junio. 26(5): 541-546.

4. Estrada M, López B. Do Thermoplastic Materials Improve the Obturation of the Root Canal? Bibliographic Review of the Different Techniques Available in the Market. J Dent Health Oral Disord Ther. 2018 January; 9(1): 00323.

5. Li G, Niu L, Zhang W, Olsen M, De-Deus G. Ability of New Obturation Materials to Improve the Seal of the Root Canal System - A Review. Acta biomaterialia. $2014 \mathrm{Dec}$; 10(3): 1050-1063.

6. Muliyar S, Shameem KA, Thankachan RP, Francis $P G$, Jayapalan CS, Hafiz KAA. Microleakage in Endodontics. Journal of International Oral Health: JIOH. 2014 Nov-Dec; 6(6): 99-104.

7. Lima M, Endodoncia - Ciencia y Tecnología. Tomo 2. Sao Paulo. Editorial Amolca; 2016.

8. Tomson RM, Polycarpou N, Tomson PL. Contemporary obturation of the root canal system. Brazilian Dental Journal. 2014 Mar; 216(6): 315-22.

9. Moeller L, Wenzel A, Wegge-Larsen A, Ding M, Kirkevang L. Quality of root fillings performed with two root filling techniques. An in vitro study using micro-CT. Acta Odontológica Scandinavica. 2013; 71(3-4): 689-696.

10. Libonati A, Montemurro E, Nardi R, Campanella V. Percentage of Gutta-percha-filled Areas in Canals Obturated by 3 Different Techniques with and without the Use of Endodontic Sealer. J Endod. 2018 March; 44 (3): 506-509.

11. Guzmán B, Koury J, García E, Méndez C, Antúnez M. Interfase Top Seal-dentina en relación con dos técnicas de obturación: condensación lateral y técnica termoplastificada/termoreblandecida. Estudio de microscopía electrónica de barrido. Univ. Odontol. 2015 Jun-Dic; 34(73): 2027-3444

12. Lima M, Endodoncia - Ciencia y Tecnología. Tomo 2. Sao Paulo. Editorial Amolca; 2016.

13. Lopes H, Siqueira J. Endodontia - Biología e Técnica. 4aㅡ ed. Sao Paulo: Ed. Elsevier; 2015.

14. Chemim H, Figueiredo W, Vinicius $M$, Carlos R. Técnicas de obturação endodónticas. Revista Faipe. 2017 June; 3(2): 30-58.

15. Salcedo D, Petkova M, Jara M, Pineda M, Donayre J, Rodríguez I. Evaluación de la calidad de obturación de la técnica de condensación vertical de Mc Spadden modificada, la técnica termoplastificada de ola continua y condensación lateral. Rev. Theorema - UNMSM. 2015; 2(2): 2735.

16. Shafer E, Schrenker C, Zupanc J, Burklein S. Percentage of Gutta-percha Filled Areas in Canals Obturated with Cross-Linked Gutta-percha Corecarrier Systems, Single-Cone and Lateral Compaction Technique. Journal Endod. 2016 February; 42(2): 294-298.

17. Li G, Niu L, Selem LC, et al. Quality of Obturation Achieved by an Endodontic Core-carrier System with Crosslinked Gutta-percha Carrier in Singlerooted Canals. Journal of Dentistry. 2014; 42(9): 1124-1134.

18. Olczak K, Pawlicka H. Evaluation of the Sealing Ability of Three Obturation Techniques Using a Glucose Leakage Test. Biomed Res Int. 2017 Jun; Volume 2017, Article ID: 2704094.

19. Vittoria G, Pantaleo G, Blasi A, Spaqnuolo G., Landolo A, Amato M. Thermafil: A New Clinical Approach Due to New Dimensional Evaluations. Open Dent Journal. 2018 Feb; 12: 173-180.

20. Emmanuel S, Shantaram K, Sushil KC, Manoj L. An in-vitro evaluation and comparison of apical sealing ability of three different obturation technique - lateral condensation, obtura II, and thermafil. Journal Int Oral Health. 2013 Apr; 5(2): 35-43.

21. Gok T, Akcay I, Davut I, Keles A. Evaluation of Different Techniques for Filling Simulated C-Shaped Canals of 3-dimensional Printed Resin Teeth. Journal Endod. Sep 2017; 43(9): 1559-1564.

22. Gupta R, Dhingra A, Panwar N. Comparative Evaluation of Three Different Obturating Techniques Lateral Compaction, Thermafil and Calamus for Filling Area and Voids Using Cone Beam Computed Tomography: An In vitro study. J Clin Diagn Res. 2015 Aug; 9(8): ZC15-ZC17.

23. Aminsobhani M, Ghorbanzadeh A, Reza M, Namjou A, Javad M. Comparison of obturation quality in modified continuous wave compaction, continuous wave compaction, lateral compaction and warm vertical compaction. Techniques. Journal Dent (Tehran). 2015 Feb; 12(2): 99-108.

24.Zoqheib C, Hanna M, Pasqualini D, Naama A. Quantitative volumetric analysis of cross-linked guttapercha obturators. Journal of Odontostomatologic Sciences. 2017 Jan; 7(3): 4651.

25. Gómez M, Valencia O, Pinilla J, Estévez R, Cisneros R. Revisión crítica actualizada sobre la obturación con el sistema thermafil y su sucesor: guttacore. Rev. Cient. Dent. 2014 Enero-Abril; 11(1): 21-31.

26. Marques-Ferreyra $M$, Abrantes $M$, Ferreyra $H-D$, Caramelo F, Botelho M-F, Carrilho E-V. Sealing efficacy of system B versus Thermafil and Guttacore obturation techniques evidenced by scintigraphic analysis. Journal of Clinical and Experimental Dentistry. 2017 Jan; 9(1): e56-e60.

27. Soo W, Thong Y, Gutman J. A comparison of four gutta-percha techniques in simulated $\mathrm{C}$-shaped 
canals. Int Endod Int Endod Journal. 2015; 48: 73646.

28. Samadi F, Jaiswal JN, Saha S, Garg N, Chowdhary $S$, Tripathi VP et al. A comparative evaluation of efficacy of different obturation techniques used in root canal treatment of anterior teeth: An in vitro study. Int J Clin Pediatr Dent. Jan-Apr; 2014; 7(1): 1-5.

29. Kerr Corporation. Soft core carrier based obturator [Internet]. 2018. [Citado: 20/12/2017]. Disponible en: https://www.kerrdental.com/soft-core-carrierbased-obturator.

30. GuttaCore. Brochure 2012 [Internet] [Citado: 15/12/2017]. Disponible en: http://www.dentsply.co.uk/uploads/files/guttacore brochure_.

31. De La Sotta F. Guttacore. Rev. Canal Abierto. Sociedad Endodóntica de Chile. 2014; 29: 42-45.

32. Jara $M$, Llanos $M$, Inga J. Comparación de la calidad de sellado de tres técnicas de obturación radicular a través del microscopio estereoscópico. Rev. Odont. S M. 2014; 17(2): 57-61.

33. Talamantes JC, Soriano PS, Camacho NE, Vásquez EM. Comparación del sellado apical: Obturación lateral con ultrasonido vs obturación con inyección de gutapercha termoplastificada. Rev. Elec. Med., Salud y Soc. 2015; 5(3): 198-208.

34. Siu E, Wei JW, Pan GS. Quality of root canal fillings using three gutta-percha obturation techniques. Restor Dent Endod. 2016 Feb; 41(1): 22-28. 